\title{
Deficiency of malate-aspartate shuttle component SLC25A12 induces pulmonary metastasis
}

\author{
H. Furkan Alkan 1,2, Paul W. Vesely ${ }^{3,4}$, Hubert Hackl ${ }^{5}$, Johannes Foßelteder ${ }^{6}$, Daniel R. Schmidt ${ }^{2,7,8}$, \\ Matthew G. Vander Heiden ${ }^{2,4,8}$, Martin Pichler ${ }^{6,9}$, Gerald Hoefler ${ }^{3,10}$ and Juliane G. Bogner-Strauss ${ }^{1,10^{*}}$ (D)
}

\begin{abstract}
Background: Aspartate biosynthesis and its delivery to the cytosol can be crucial for tumor growth in vivo. However, the impact of intracellular aspartate levels on metastasis has not been studied. We previously described that loss-of-aspartate glutamate carrier 1 (SLC25A12 or AGC1), an important component of the malate-aspartate shuttle, impairs cytosolic aspartate levels, $\mathrm{NAD}^{+} / \mathrm{NADH}$ ratio, mitochondrial respiration, and tumor growth. Here, we report the impact of AGC1-knockdown on metastasis.

Results: Low AGC1 expression correlates with worse patient prognosis in many cancers. AGC1-knockdown in mouse lung carcinoma and melanoma cell lines leads to increased pulmonary metastasis following subcutaneous or intravenous injections, respectively. On the other hand, conventional in vitro metastasis assays show no indication of increased metastasis capacity of AGC1-knockdown cells.

Conclusion: This study highlights that certain branches of metabolism impact tumor growth and tumor metastasis differently. In addition, it also argues that commonly known metastasis indicators, including EMT genes, cell migration, or colony formation, do not always reflect metastatic capacity in vivo.
\end{abstract}

Keywords: Malate-Aspartate Shuttle, AGC1, Aralar, SLC25A12, Metastasis, Aspartate

\section{Introduction}

Tumor metastasis is correlated with poor prognosis. Surgical removal of the metastatic tumors from one or more organs is challenging, and drug-resistance, induced by the new tissue environment, may occur in metastatic tumors [1]. To form metastasis, cancer cells follow a cascade of events including invading the surrounding tissue, detaching from the primary tumor, surviving in the circulation, and colonizing a distant organ $[2,3]$. In addition to the bioenergetics and biosynthetic demands of cell proliferation,

\footnotetext{
* Correspondence: juliane.bogner-strauss@stmk.gv.at

${ }^{1}$ Institute of Biochemistry, Graz University of Technology, Humboldtstrasse

46/III, 8010 Graz, Austria

${ }^{10}$ BioTechMed-Graz, Graz, Austria

Full list of author information is available at the end of the article
}

metastatic cells also need to adapt their metabolism to succeed in the different steps of this cascade of events [1]. Although a definitive metabolic pattern that distinguishes metastatic from non-metastatic tumors has not yet been identified, there is evidence that certain metabolic pathways could be more important for metastasis formation [2]. For instance, antioxidants such as $\mathrm{N}$-acetylcysteine (NAC) increase melanoma metastasis in mice [3, 4], suggesting that suppressing oxidative stress is one requirement of distant metastasis. Consistently, inhibition of the folate pathway using methotrexate or knockdown of either ALDH1L2 (aldehyde dehydrogenase 1 family member L2) or MTHFD1 (methylenetetrahydrofolate dehydrogenase) blocks melanoma metastasis in vivo via 
hindering the survival of circulating melanoma tumors while having no impact on subcutaneous tumor growth [5]. Similarly, lactate uptake through MCT1 (monocarboxylate transporter 1) expression has no effect on primary tumor growth but improves the number of circulating tumor cells and promotes distant melanoma metastasis [6]. In addition, proline catabolism is selectively important for the proliferation and colony formation of metastatic cells, and reduced Prodh (proline dehydrogenase) activity leads to impaired in vivo lung metastasis in mouse breast cancer tumors [7]. Pyruvate was also shown to be an important mediator of metastatic niche formation through hydroxylation of collagen [8]. Furthermore, asparagine levels or ASNS (asparagine synthetase) activity could also determine the metastatic capacity of breast cancers both in vivo and in vitro [9].

Aspartate is a proteinogenic non-essential amino acid that is used for de novo synthesis of nucleotides and certain non-essential amino acids. Aspartate is also involved in several intracellular processes including, but not limited to, redox homeostasis and the urea cycle. Intracellular aspartate levels correlate with the proliferation rate in cancer cells [10] and are limiting for the growth of some tumors in vivo [11, 12]. Although aspartate is available in circulation, these levels are insufficient to trigger its uptake in most cells that lack the plasma membrane aspartate transporter SLC1A3 [11]. Therefore, endogenous aspartate synthesis can limit the growth of some tumors. For example, one requirement for aspartate production is a suitable $\mathrm{NAD}^{+} / \mathrm{NADH}$ ratio. Cells lacking mitochondrial respiration have low aspartate levels and slow proliferation [13, 14]. In addition, glutamine is an important substrate for mitochondrial aspartate synthesis. In our previous study, we determined that blocking glutamine metabolism and/or inhibiting cytosolic aspartate delivery via knockdown of mitochondrial aspartate-glutamate carrier 1 (AGC1) impairs cell proliferation and tumor growth [15]. Altogether, our previous findings suggest that not only having the adequate conditions (high $\mathrm{NAD}^{+} / \mathrm{NADH}$ ratio) and the substrate (glutamine) available for aspartate synthesis but also the ability of the mitochondria to export its aspartate to the cytosol can be a limitation for cell proliferation [16].

In this follow-up study, we report that AGC1 knockdown (KD) in mouse Lewis lung carcinoma (LLC1) cells leads to increased lung metastasis and poor overall survival of syngeneic mice, despite impaired subcutaneous tumor growth. Similarly, we observed enhanced metastatic spread of intravenously injected B16F10 cells upon AGC1-KD. Interestingly, conventional in vitro metastasis assays show no indication of increased metastasis capacity of AGC-KD cells, suggesting that the role of aspartate metabolism in metastasis formation is relevant to metastatic events occurring only in vivo. Collectively, our report argues that certain branches of metabolism impact tumor growth and tumor metastasis differently.

\section{Materials and methods Bioinformatics}

Gene expression profiles (RNA sequencing V2 data) of SLC25A12 (Aralar or AGC1) and SLC25A13 (Citrin or AGC2), as well as clinical information for 21 different cancer type cohorts of The Cancer Genome Atlas (TCGA) were retrieved via firebrowse.org (courtesy Broad Institute of MIT \& Harvard). Using the clinical and expression data from TCGA database, survival analyses were performed on dichotomized groups of patients with high and low expression levels using an optimized cutoff according to the maximal Harrels concordance index. The Kaplan-Meier method was used to estimate survival distributions and the log-rank test was used to evaluate statistical significance in overall survival (OS) between patient groups. Where stated, $p$ values were adjusted for multiple testing as described previously [17]. Log2 hazard ratios and 95\% confidence interval were visualized as forest plot. Negative log2 hazard ratios represent gene expressions correlating with patient survival. Differences in gene expression of the primary tumor between pathological metastatic (M) stages were tested using the non-parametric two-sided Wilcoxon rank sum test and visualized as boxplots. All analyses were performed using $\mathrm{R}$ and packages survival and dynpred.

\section{Cell culture}

All cultured cells tested negative for mycoplasma. All cell lines were maintained in DMEM $(25 \mathrm{mM}$ glucose, 4 $\mathrm{mM}$ glutamine, $1 \mathrm{mM}$ sodium pyruvate) supplemented with $10 \%$ fetal bovine serum (FBS, Gibco) and 50 units/ $\mathrm{mL}$ penicillin/streptomycin (Gibco) in sterile incubators set to $37^{\circ} \mathrm{C}$ temperature and $5 \% \mathrm{CO}_{2}$ concentration. AGC1 protein was knocked-down using shRNA as described previously [15].

\section{Scratch assay}

$6 \times 10^{5}$ cells were seeded in a 6-well plate and incubated for $24 \mathrm{~h}$. Cells were harvested by trypsinization, and $1 \times$ $10^{5}$ transfected cells were seeded in a 24-well culture plate in 6 replicates and incubated for $24 \mathrm{~h}$ to form a confluent cell monolayer. The scratch was introduced using a $200 \mu \mathrm{L}$ pipette tip, and detached cells were washed off with PBS.

Cells were further incubated with fresh growth media, and scratch closure was observed using an inverted light microscope (Olympus IX71). The scratch width was 
measured using the cellSens imaging software (Olympus) every $4-12 \mathrm{~h}$ depending on the cell line. Arithmetic mean of all replicates was used to calculate the scratch closure of cells.

\section{Sphere formation assays}

To test the ability of cells with modified gene expression levels to form tumor spheres, we generated tumor spheres as previously described [18]. In detail, the adherent growing cell lines were dissociated into single cells using trypsin/EDTA and 2000 single cells per well seeded in ultra-low attachment 6-well plates (Corning, NY, USA) using serum-free MEBM (Lonza, Basel, Switzerland) medium (SFM) supplemented with 1xB27 supplement (Gibco), $20 \mathrm{ng} / \mathrm{ml}$ human epidermal growth factor EGF (Peprotech, Hamburg, Germany), $10 \mathrm{ng} / \mathrm{ml}$ human basic fibroblast growth factor FGF (Peprotech), $20 \mathrm{IU} / \mathrm{ml}$ heparin (Baxter, Vienna, Austria), and 1\% antibiotic/antimycotic solution (Thermo Fisher Scientific, containing 10,000 units/mL of penicillin; $10,000 \mu \mathrm{g} / \mathrm{mL}$ of streptomycin; and $25 \mu \mathrm{g} / \mathrm{mL}$ of Gibco Amphotericin B). After 14 days, the number of spheres was counted using a bright-field microscope in three independent replicates.

\section{Migration Assay}

Five thousand cells in $100 \mu \mathrm{L}$ DMEM containing 1\% FBS were seeded into insert wells of the Transwell plates (Corning). Lower wells were filled with $500 \mu \mathrm{L}$ DMEM containing 10\% FBS. Forty-eight hours later, cell numbers in both upper and lower wells were measured via either conventional cell counting or using Cell Titer Glo (Promega) as described previously [15].

\section{ROS measurement}

Sub-confluent LLC1 cells were incubated with $1 \mu \mathrm{M}$ CellRox (RED2 Channel, Invitrogen) solution for $1 \mathrm{~h}$, washed twice with PBS, trypsinized, and resuspended with media by pipetting long enough to avoid cell clumps. After centrifugation, cells were mixed well with $100 \mu \mathrm{L}$ PBS and incubated with Ghost Dye (1:1000 dilution; NIR2 Channel, Tonbobio) for half an hour. Ghost Dye was washed away, and cells were resuspended in PBS. Fluorescent signals were obtained via Guava Easy Cyte 8 (Millipore), and data were analyzed using the InCyte Software (Millipore).

\section{Animal care}

The animal study was approved by the institutional ethics committee, and experiments were performed according to the guidelines of the Austrian Federal Ministry of Science and Research. Experiment licenses were granted under BMWF-66.007/0026-WF/V/3b/ 2015 and BMWF-66.007/0008-WF/V/3b/2016. In vivo,
CB-839 experiments were approved by the MIT Committee on Animal Care (IACUC).

\section{LLC1 subcutaneous tumor growth}

LLC1 cells were cultured in T175 flasks about a week prior to the day of injection. For getting ready with the injections, cells were grown to 50-60\% confluency, washed with $\mathrm{PBS}$, mixed with $5 \mathrm{~mL}$ trypsin and resuspended with $25 \mathrm{~mL}$ media as soon as they detach from the plate. Cells were passed through $0.45 \mathrm{~mm} \times 25 \mathrm{~mm}$ needles to eliminate clumps and centrifuged for $5 \mathrm{~min}$ at $800 \mathrm{~g}(1000 \mathrm{rpm})$. Media was aspirated; the pellet was resuspended with $3 \mathrm{~mL}$ ice-cold HBSS; viable cells were counted using a hemocytometer and diluted to $2.5 \times 10^{6}$ cells $/ \mathrm{mL}$ in HBSS. Cells were kept at $4{ }^{\circ} \mathrm{C}$ and used for injection within less than $2 \mathrm{~h}$. Using $0.6 \mathrm{~mm} \times 30 \mathrm{~mm}$ needles, $0.3 \times 10^{6}$, or $0.5 \times 10^{6}$ cells (in $200 \mu \mathrm{L}$ ) were injected over the right flanks or behind the neck of 8week-old female C57BL/6 mice (8 mice per group), respectively. Mice were sacrificed at day 16 or day 21, and primary (subcutaneous) tumors and lung tissues were collected for further analysis. The survival rate of the mice was monitored at least once every day in standard housing conditions ensuring their access to water and food supply. CB-839 treatments were performed as described previously [15].

\section{B16F10 intravenous injections}

B16F10 cells were cultured and prepared for injection as it is described above for LLC1 cells. Cells were diluted to $3 \times 10^{6}$ cells $/ \mathrm{mL}$ in cold HBSS. 0.3 $\times 10^{6}$ cells $(100 \mu \mathrm{L})$ were injected into the tail vein of 8-10 week-old female C57BL/6 mice using $0.3 \mathrm{~mm}$ $\times 12 \mathrm{~mm}$ needles (Omnican). The mice were harvested at day 16 , and lungs were collected, rinsed, and fixed in $10 \%$ formalin for at least $48 \mathrm{~h}$.

\section{Tumor area measurements in the lung}

For pulmonary tumor area measurement, each lung was sliced into 12-15 pieces, embedded in paraffin, stained with hematoxylin and eosin (H\&E), and total area, as well as tumor area, was measured for every piece using the NisElements software. In addition, number of tumor loci was counted, and individual sizes of each tumor locus was recorded. Tumors were determined using $x$ 10 objectives; areas were measured using $\times 2$ objectives.

\section{Immunohistochemistry}

$\mathrm{IHC}$ in lung tissues of mice bearing B16F10 tumors was performed as described previously [19]. Briefly, formalin-fixed paraffin-embedded lung tissue was prepared as described for lung area measurement. We used anti-PCNA antibody (Chemicon-MAB424, 1:2500 dilution). Antibody binding was visualized using AEC 
(cat\#3464, Dako, Glostrup, Denmark). Tissue samples were counter stained with hematoxylin according to standard methods.

IHC in lung tissues of mice bearing LLC1 tumors was performed by the Histopathology Core at the Koch Institute's Robert A. Swanson Biotechnology Center using a Thermo Scientific LabVision 360 autostainer. Antigen retrieval was performed at $97^{\circ} \mathrm{C}$ for $20 \mathrm{~min}$ in pH 6 citrate buffer (Abcam \#3678). Slides were blocked using Rodent Block M (Biocare Medical \#RBM961L). Primary antibodies were Cleaved Caspase 3 (CC3; Cell Signaling Technologies \#9664 L, 1:800 dilution) and Ki67 (Biocare Medical CRM 325B, 1:50 dilution). Secondary detection reagents were Mouse on Mouse HRP (Biocare Medical \#MM620L) and Rabbit on Rodent HRP (Biocare Medical \#RMR622L). After incubation with DAB Quanto Chromogen Substrate (ThermoScientific \#TA-125-QHDX), slides were counterstained and scanned with Leica Aperio AT2.

\section{Quantitative real-time PCR}

Total RNA from cells was isolated using the PeqGOLD total RNA isolation kit (Peqlab). Tissue RNA was isolated with TRIzol reagent (Life Technologies). cDNA was generated using the Qiagen QuantiTect RT kit. mRNA expression was assessed using SYBR green realtime PCR on an ABI 7000 instrument as described. Unless stated otherwise, $\mathrm{Ct}$ values of every gene was first subtracted from the ones of Rplp0 (for LLC1 cells) and TfIIb (for B16F10 cells) within every cDNA sample, and then normalized to the mean $\delta \mathrm{Ct}$ of the control group for every gene. Fold changes were calculated by taking the $\delta \delta \mathrm{Ct}$ power of $(1 / 2)$.

\section{Statistical analysis}

All experiments were performed using at least three independent replicates (different passages and mice for in vitro and in vivo experiments, respectively). Single comparison between two groups, is calculated via two-tailed, unpaired Student's $t$ test. Significance levels: ${ }^{*} p \leq 0.05,{ }^{* *} p \leq 0.01$, **** $p \leq 0.001$.

\section{Results}

Low AGC1 expression correlates with worse prognosis in some cancers

We previously generated shRNA-mediated knockdowns (KD) of mitochondrial aspartate-glutamate carrier 1 (AGC1) and observed that AGC1-KD inhibited Lewis Lung Carcinoma (LLC1) tumor growth [15]. However, mice with AGC1-KD tumors tended to die earlier than controls, even though their tumors were smaller (Fig. 1a, b). This implies that AGC1 might have an impact on cancer beyond affecting cell proliferation and tumor growth. Analysis of overall patient survival and gene expression from The Cancer Genome Atlas (TCGA) showed that human lung adenocarcinoma tumors (LUAD) with lower AGC1 (SLC25A12) mRNA expression correlated with worse prognosis (Fig. 1c). Although this correlation was not universal across all cancer types analyzed, several other cancer types including cervical squamous cell carcinoma (CESC), kidney renal clear cell carcinoma (KIRC), kidney renal papillary cell carcinoma (KIRP), and sarcoma (SARC) showed a similar association between AGC1 expression and overall patient outcome (Fig. 1d). Interestingly, the correlation between expression of the functionally identical AGC2 (SLC25A13) isoform [20] and patient survival was usually in the opposite direction of AGC1 within the same type of cancer (Fig. 1d). Only in kidney renal cell carcinomas, low expression levels of both AGC1 and AGC2 showed a distinct correlation with the poor prognosis of patients (Fig. 1d, e). Further bioinformatics analysis of this type of cancer suggested mRNA expressions of both AGC1 and AGC2 were lower in metastatic regions compared to the primary tumors (Fig. 1f), implying that AGC1 might influence metastatic spread in human cancers.

\section{AGC1-KD increased the pulmonary metastasis from LLC1 and B16F10 cells}

Based on the bioinformatics analyses and because metastasis is the leading cause of death in cancer patients, we hypothesized that AGC1-deficient cells might have a superior metastatic capacity compared to control cells. Thus, we performed a second line of subcutaneous LLC1 tumor cell injections and assessed the lungs of the mice at the end of the experiment. Strikingly, histological analysis showed that none of the mice from the control group had pulmonary metastasis while approximately half of the mice bearing AGC1-KD tumors had evidence of lung metastasis (Fig. 2a). To achieve bigger primary tumors and more metastasis, we next injected LLC1 cells subcutaneously behind the neck of the mice instead of the flanks. This approach allows injecting higher number of cells and a long period of tumor growth. Eventually, we sacrificed the mice and harvested the lungs 21 days after tumor cell injection, and the percentage of tumor area within the lung was measured by morphometric analysis. We observed the percentage of metastatic area within the lungs from the mice bearing AGC1-KD LLC1 tumors to be significantly higher than those from mice bearing control tumors (Fig. 2b). In addition, we counted the number of discrete metastatic tumors in the lungs and observed that AGC1-KD cells produced a greater number of tumor loci in the lungs compared to controls (Fig. 2c). However, the average size of each individual tumor loci that formed was not significantly 
A.

Tumor Growth

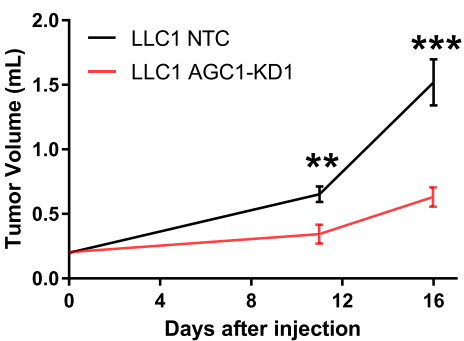

C.

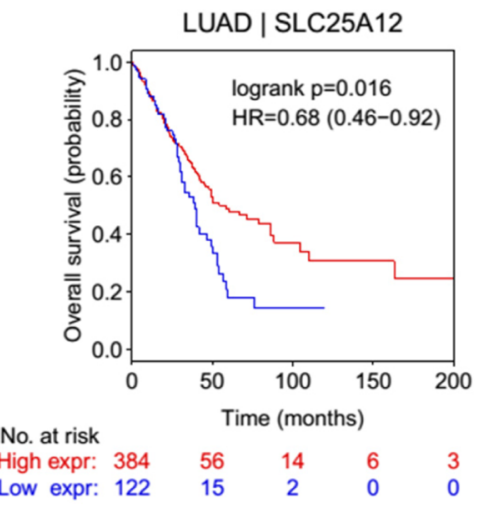

E.

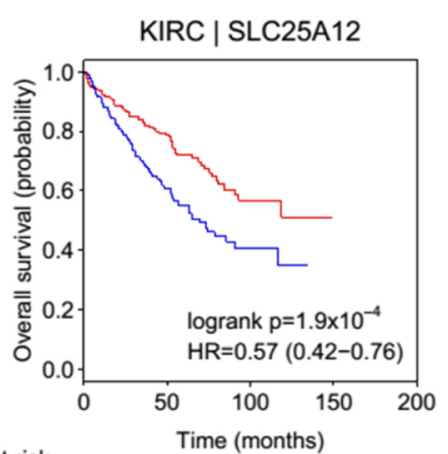

No. at risk

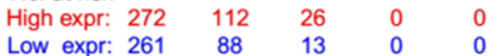

F.

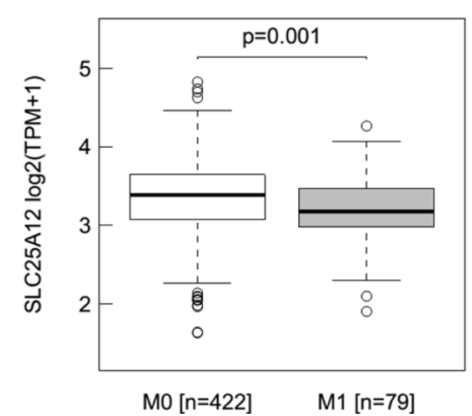

$\mathrm{M} 0[\mathrm{n}=422] \quad \mathrm{M} 1[\mathrm{n}=79]$
B. Survival Rates

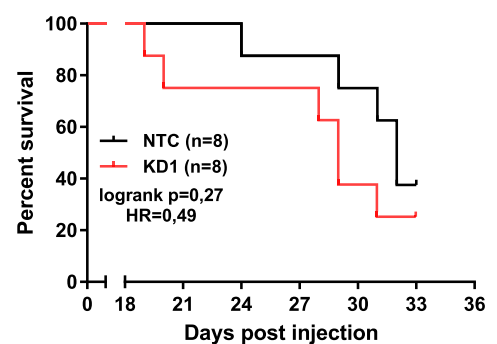

D.

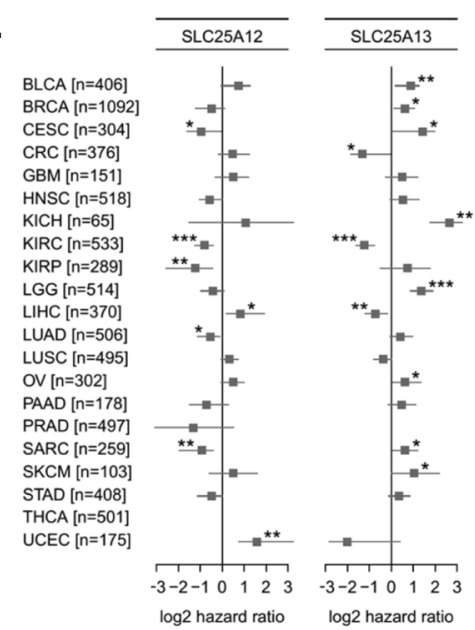

KIRC | SLC25A13

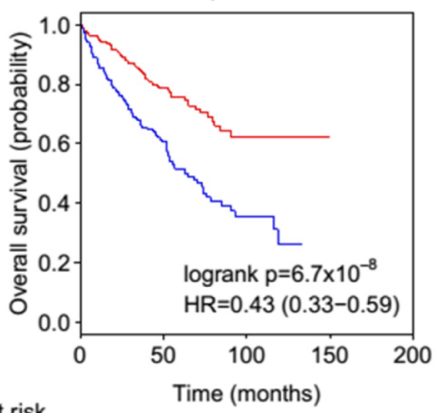

No. at risk

High expr: $\begin{array}{ccccc}272 & 110 & 24 & 0 & 0 \\ 261 & 90 & 15 & 0 & 0\end{array}$
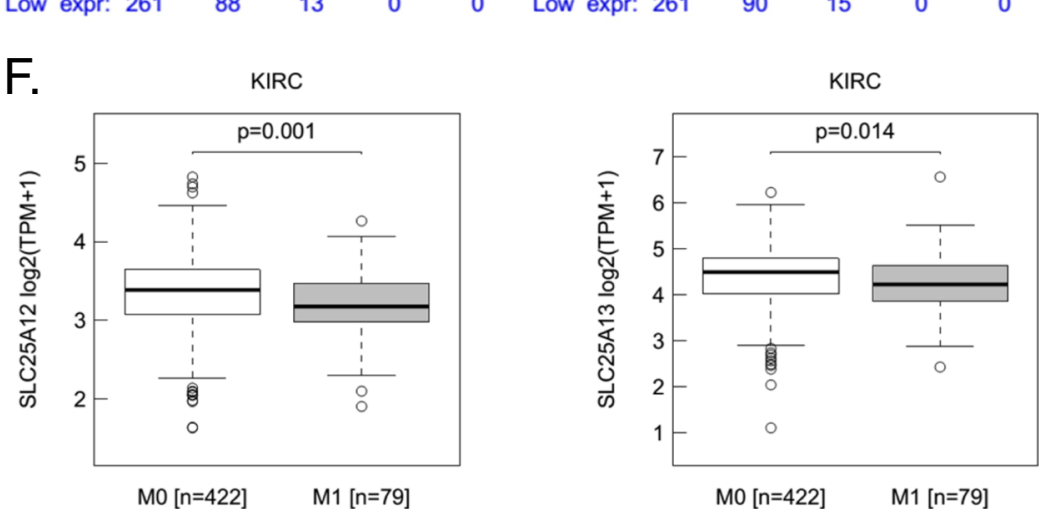

Fig. 1 (See legend on next page.) 
(See figure on previous page.)

Fig. 1 AGC1-deficient tumors tend to be more lethal for mice. a Tumor progression of control (NTC) and AGC1-KD LLC1 tumors measured as described above. $\mathbf{b}$ Survival rates of the mice bearing control (NTC) and AGC1-KD LLC1 tumors monitored over the course of 33 days. c Overall survival rates of patients with high (red line) or low (blue line) AGC1 (SLC25A12) mRNA-expressing lung adenocarcinomas (LUAD). Data is adopted from the TCGA database. Patients with higher or lower AGC1 (SLC25A12) mRNA expression were divided into two groups with a cutoff line where the separation of these groups was significantly most meaningful. d Correlation of AGC1 (SLC25A12) or AGC2 (SLC25A13) mRNA expression with better (negative $\log _{2}$ hazard ratio) or poor (positive $\log _{2}$ hazard ratio) survival of patients from various cancers. Lower log 2 hazard ratio for a gene means that the group of patients with low expressing tumors have worse overall survival than the high-expressing group. The cutoff to separate groups was determined as described in c. e Overall survival (OS) rates of patients with high (red line) or low (blue line) AGC1 (SLC25A12) or AGC2 (SLC25A13) mRNA-expressing kidney renal clear cell carcinoma tumors (KIRC). Data is adopted from the TCGA database. The cutoff to separate groups was determined as described in c. f mRNA expressions of AGC1 (SLC25A12) or AGC2 (SLC25A13) in kidney renal clear cell carcinoma tumors (KIRC) of patients without (M0) or with metastasis (M1)

different between mice bearing AGC1-KD tumors compared to control tumors (Fig. 2c). These data may suggest that the number of cells seeded to the lung, rather than the rate of tumor growth one cell seed the lung may account for the increased metastatic tumor area observed upon AGC1-KD. Because AGC1-KD enhances sensitivity of primary tumors to glutaminase inhibition [15], we tested whether mice treated with the glutaminase inhibitor CB-839 would have a different metastasis outcome. However, inhibiting glutamine metabolism in vivo had no impact on metastasis of either control or AGC1-KD tumors (Fig. 2d). Finally, we examined both apoptosis and proliferation markers in metastatic AGC1-KD and control lesions. Both AGC1-KD and control metastatic tumors exhibited relatively higher Ki-67 staining (marker of proliferation) than staining for Cleaved Caspase 3 (CC3, marker for apoptosis), but staining for both was comparable between AGC1-KD and control tumors (Fig. 2e). These data suggest that AGC1-KD tumors may survive better in circulation compared to control tumors or improve tumor initiation in the metastatic site, which leads to an increased metastatic burden in the lung.

To test whether AGC1-loss also increases metastasis formation in a different cancer model, we knockeddown AGC1 in B16F10 mouse melanoma cell line that is specifically generated for their metastatic potential [21]. AGC1 depletion in B16F10 cells reduced cell proliferation to a comparable extent as in LLC1 cells and in other cell lines from our previous study (Figure S1A) [15]. To test the impact of AGC1-knockdown on the metastatic spread of B16F10 cells in vivo, we injected them intravenously in mice. Sixteen days post-injection, mice were sacrificed, lungs were harvested, and the percentage of tumor area within the lung was determined as described above. Strikingly, AGC1-KD B16F10 cells also yielded more metastatic areas in the lungs compared to control cells (Fig. 3a, b, S1B). These findings suggest that AGC1-loss is correlated with increased metastatic capacity in independent cancer models. Although the number of metastatic tumor loci found in mice bearing AGC1-KD tumors was not significantly different than mice bearing control tumors, there was a similar trend toward an increased number of foci in B16F10 AGC1-KD bearing mice (Fig. 3c). However, the sizes of tumor loci were variable, which may be explained by multiple tumor loci merging to appear as a single locus as they grow larger (Fig. 3b, S1B). Importantly, size of the individual tumors (Fig. 3c) and the expressions of proliferation and apoptosis markers (Fig. 3d, Figure S2) were not increased in metastatic AGC1-KD tumors compared to control counterparts, suggesting that an increased chance of survival in circulation or an increase in the efficiency of metastatic tumor initiation might account for the enhanced metastatic capacity of AGC1-KD B16F10 cells.

\section{Increased metastatic capacity of AGC1-KD cells cannot be detected by in vitro methods}

The finding that the AGC1-KD tumor cells show relatively slow proliferation but high metastatic potential led to the conclusion that biological events other than proliferation rate are likely to cause this phenomenon. Hence, we performed a series of in vitro tests to analyze migration speed and colony formation capacity. First, we performed a scratch (wound-healing) assay to assess the ability of AGC1KD cells to migrate. However, we found that AGC1KD LLC1 and B16F10 cells covered the scratched area slower compared to corresponding control cells (Fig. 4a, b). Because the outcomes from this experiment may be due to the reduced proliferation rate of AGC1-KD cells, we next performed migration assays using trans-well plates. We normalized the number of cells that migrated into the bottom plate to the proliferation rate and still found that the migration rate of AGC1-KD LLC1 cells was almost identical to those of control cells (Fig. 4c). Next, we measured three-dimensional tumor sphere formation ability of AGC1-KD cells. Consistent with proliferation, migration, and wound-healing assays, AGC1-KD cells showed a reduced capacity to form spheres under low-attachment conditions (Fig. 4d, e). Only in 
A.

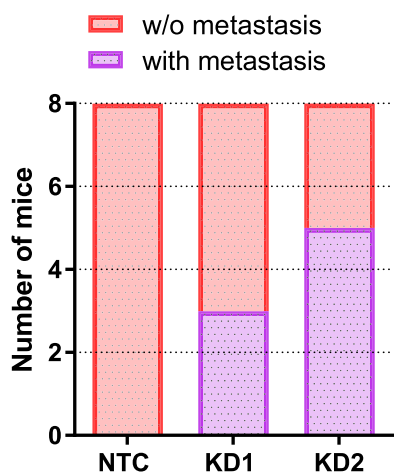

C.
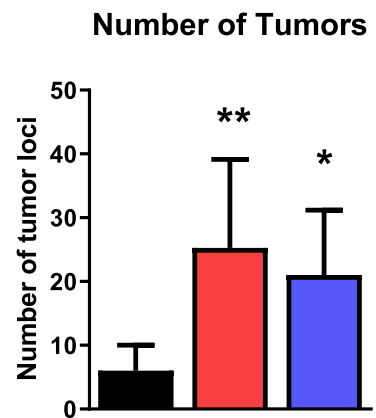

D.

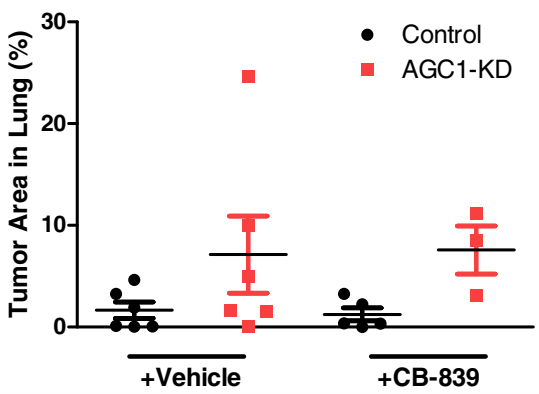

E.

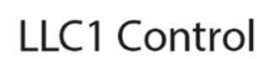

CC3
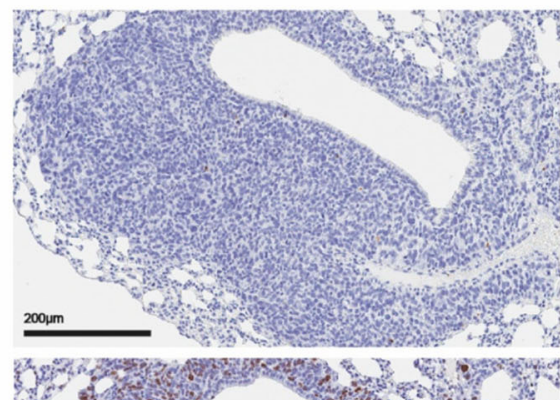

3.
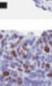

Ki67

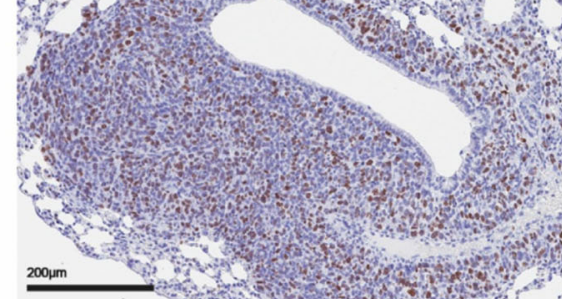

Average size of tumors
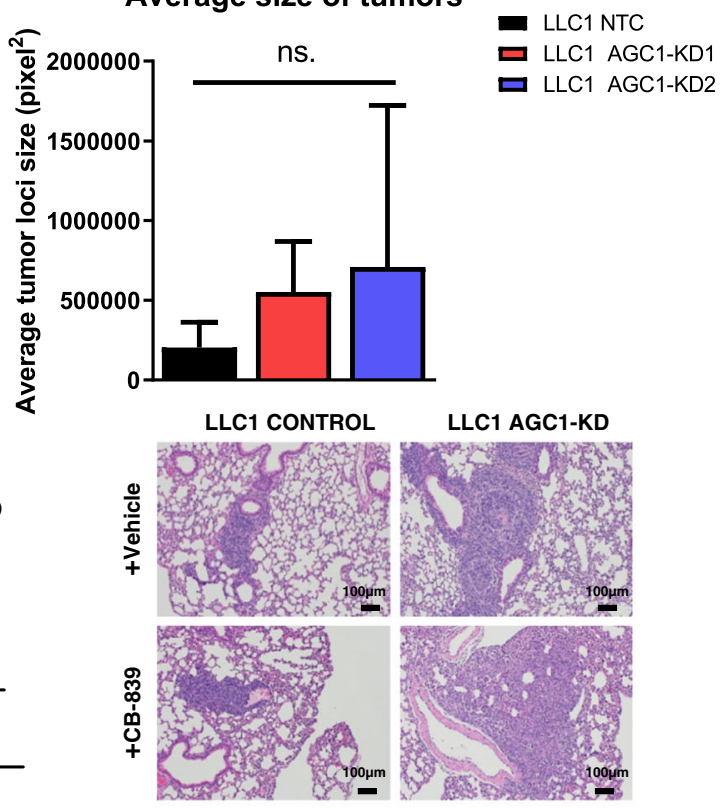

LLC1AGC1-KD1

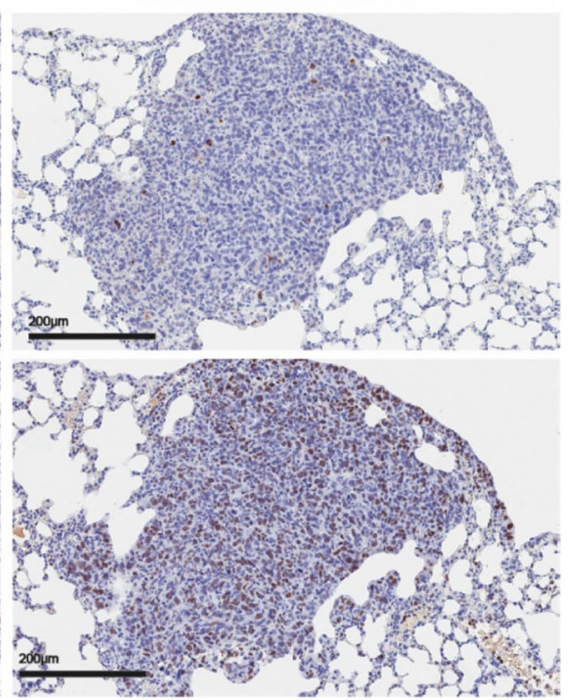

Fig. 2 (See legend on next page.) 
(See figure on previous page.)

Fig. 2 Lewis lung carcinoma (LLC1) cells with AGC1-knockdown have higher potential to metastasize. a The number of mice with (purple) or without (pink) lung metastasis 16 days after control (NTC) or AGC1-KD LLC1 cells (KD1, sh911 or KD2, sh908) were injected in the flanks. H\&Estained lung slides were screened for metastasis. $\mathbf{b}$ Percent of the metastatic area in the lungs of mice bearing control (NTC), or AGC1-KD (KD1 with sh911; KD2 with sh908) LLC1 tumors, measured 21 days after cells were injected subcutaneously behind the necks of mice $(n \geq 6)$. Lungs were resected, and H\&E-stained sections were analyzed. c (left) Number of metastatic tumor loci and (right) average tumor locus size in the lungs of mice bearing control (NTC), or AGC1-KD (KD1 with sh911; KD2 with sh908) LLC1 tumors, measured 21 days after cells were injected subcutaneously behind the necks of mice $(n \geq 6)$. Lungs were resected and H\&E-stained sections were analyzed. $\mathbf{d}$ (left) Percent of the metastatic area in the lungs of mice that were bearing control (NTC), or AGC1-KD LLC1 tumors on the flanks 22 days after injections. Mice were treated without (vehicle) or with CB-839 dosed at $200 \mathrm{mg} / \mathrm{kg}$ twice daily starting on day 13 ( $n \geq 6$ ). (right) Representative histology pictures of the metastatic areas of mice injected with control (NTC) or AGC1-KD LLC1 tumors. e Representative images of the metastatic areas of vehicle-treated mice (as in d) injected with control (NTC) or AGC1-KD LLC1 tumors, stained with proliferation (Ki67) and apoptosis (cleaved caspase 3; CC3) markers. Images were taken at $\times 20$ magnification. All experiments denote mean \pm SDs. Significance levels: ${ }^{*} p \leq 0.05,{ }^{* *} p \leq 0.01,{ }^{* * *} p \leq 0.001$

B16F10 cells, the sizes of the tumor spheres of AGC1-KD cells were comparable to the colony sizes of the control cells, although this also did not explain the increased metastatic phenotype observed in vivo (Fig. 4f, g). In summary, AGC1-KD cells showed no evidence of improved metastatic capacity using common surrogate assays for metastatic cell properties in vitro.

\section{Genes related to Aldh111 metabolism are deregulated in AGC1-KD cells}

Epithelial-to-mesenchymal transition (EMT) is an important hallmark of cancer metastasis. Accordingly, it is thought that it is a pre-requisite for cancer cells to switch from an epithelial to a mesenchymal cell surface phenotype to allow for escape from the primary tumor tissue mass [2]. To test whether LLC1 tumors with AGC1-KD show an enhanced mesenchymal phenotype compared to controls, we measured mRNA expressions of common EMT and stemness marker genes in AGC1KD tumors. Snai1, Snai2, Sox2, Skp2, and Twist1 were previously reported to induce EMT and metastasis, and E-cadherin expression is reversely correlated with EMT $[22,23]$. None of these markers, however, showed expression differences between AGC1-KD and control groups (Fig. 5a). According to this definition, LLC1 AGC1-KD tumors did not show an increased EMT phenotype compared to controls.

As a mediator of cellular oxidative stress, folate metabolism can be another important driver that dictates metastatic capacity $[5,24]$. Therefore, we measured gene expression levels of folate pathway enzymes in LLC1 tumor and cultured cells. Aldh1l1 and Aldh112 mRNA levels were consistently lower in AGC1-KD tumors and cultured cells compared to corresponding controls (Fig. 5a, b), suggesting that folate pathway might be deregulated upon AGC1-loss. In addition, intracellular reactive oxygen species (ROS) levels were significantly lower in AGC1-KD LLC1 cells (Fig. 5c). This is consistent with potentially altered folate metabolism. Because suppressing oxidative stress can assist the survival of circulating cells [5], lower baseline levels of ROS might be one explanation why AGC1-depletion has a positive impact on metastatic capacity. However, B16F10 cells showed no difference in ROS levels (Fig. 5d) or folate-pathway genes upon AGC1-KD (Fig. 5e) despite enhanced metastatic spread capacity (Fig. 3a), arguing other pathways must also be involved (Fig. 5f).

An ability to escape the immune response is important for cancer cells to survive in circulation and form metastasis in distant organs $[25,26]$. In our previous study, we found that AGC1-KD cells have lower cytosolic $\mathrm{NAD}^{+} /$ $\mathrm{NADH}$ ratio and are less glycolytic [15]. Because inflammatory cells are often more glycolytic than antiinflammatory cells [27], we speculated that AGC1-KD cells might have an advantage to evade immune response by secreting anti-inflammatory cytokines. However, mRNA levels of tested cytokines were not significantly altered in AGC1-KD LLC1 tumors compared to the controls, although some anti-inflammatory cytokines such as interleukin-10, granulocyte-macrophage colonystimulating factor (GM-CSF), and transforming growth factor beta (TGF- $\beta$ ) showed tendencies towards upregulation in AGC1-KD tumors (Figure S3A). On the other hand, findings from the in vitro experiment and B16F10 cells were again inconsistent with this observation in LLC1 cells (Figure S3B, S3C). These findings argue that contributions of many pathways, or an unknown mechanism, are responsible for the metastatic capacity of AGC1-KD tumors in vivo.

\section{Discussion}

In this study, we report that reduced mitochondrial aspartate export and malate-aspartate shuttle activity boost pulmonary metastasis of mouse lung (LLC1) and melanoma (B16F10) cell lines in vivo, despite slowing down cell proliferation and primary tumor growth [15]. To our knowledge, this is the first report indicating that impaired aspartate metabolism, which is a limiting metabolite for tumor growth, increases the metastatic potential of cancer. Furthermore, this 
A.

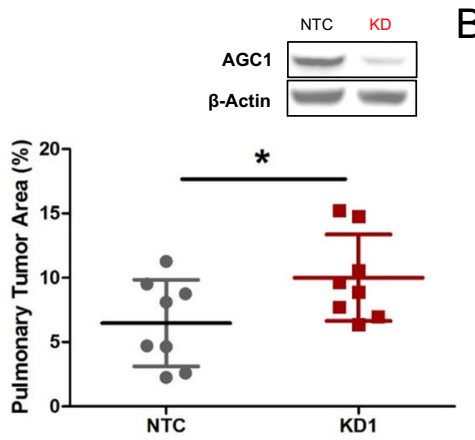

B.

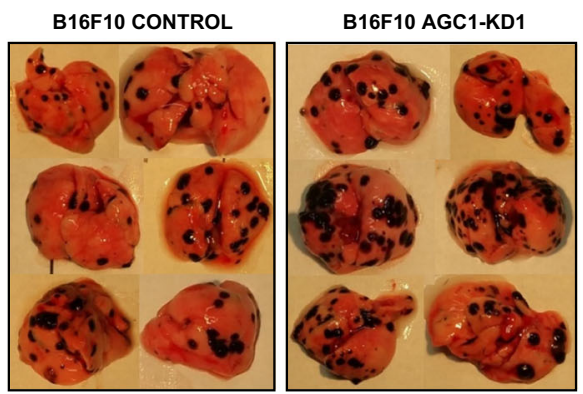

C.

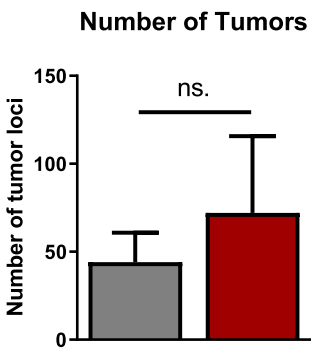

Average size of tumors

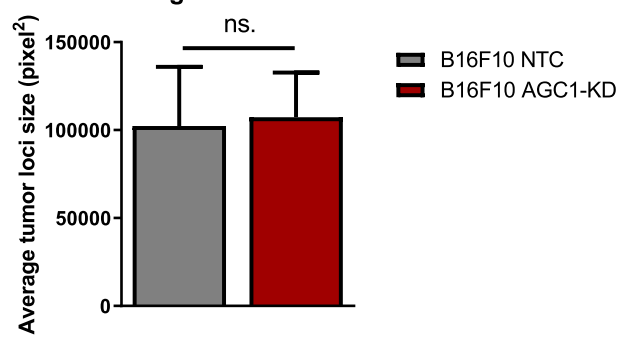

D.

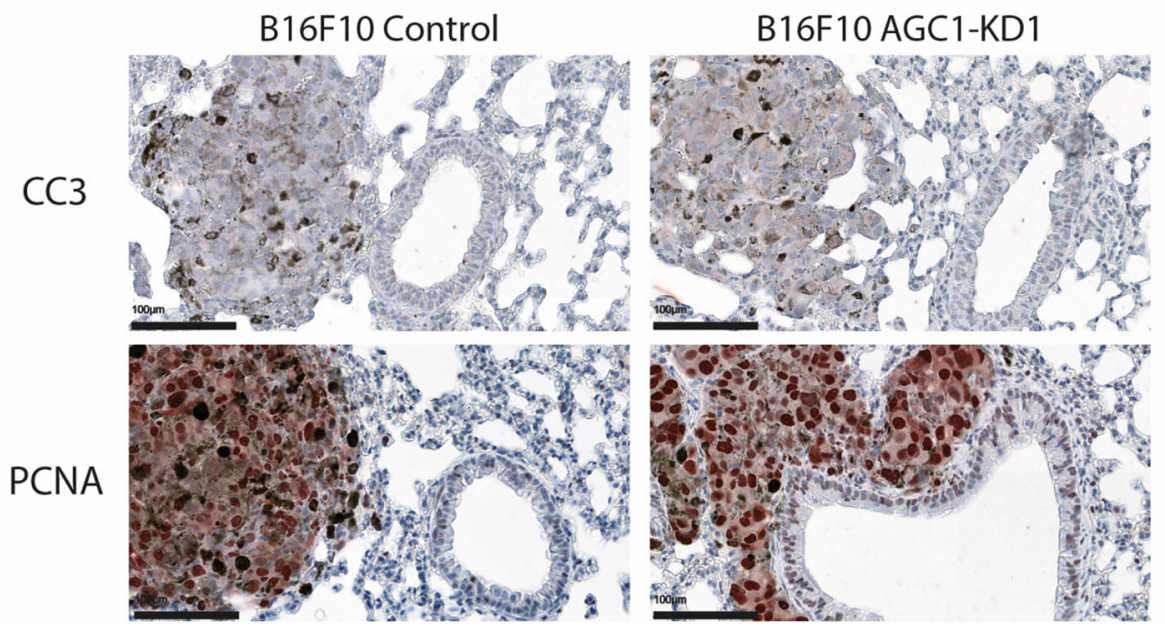

Fig. 3 B16F10 mouse melanoma cells with AGC1-knockdown have higher potential to metastasize. a Percent of the metastatic areas (\%) in the lungs of mice with control (NTC), or AGC1-KD B16F10 cells, measured 16 days after intravenous injection of cells $(n=8)$. $\mathbf{b}$ Representative images of lungs with metastasis harvested 16 days after control (NTC), and AGC1-KD B16F10 cells were intravenously injected. c (left) Number of metastatic tumor loci and (right) average tumor locus size in the lungs of mice with control (NTC), or AGC1-KD B16F10 cells, measured 16 days after intravenous injection of the cells $(n=8)$. $\mathbf{d}$ Representative images of the metastatic areas of mice injected with control (NTC) or AGC1-KD B16F10 tumors, stained with proliferation (PCNA) and apoptosis (cleaved caspase 3; CC3) markers. Images were taken at $\times 40$ magnification. All experiments denote mean \pm SDs. Significance levels: * $p \leq 0.05$

report highlights that commonly known metastasis indicators, including EMT genes, cell migration, or colony formation, do not always reflect the metastatic capacity in vivo, and our study provides strong evidence that metabolic requirements of metastasis may be different from proliferation itself.

It is important to note that the exact mechanism of how the loss of AGC1 induces pulmonary metastasis is not fully explained in this study. Altered levels of folate pathway genes, reduced intracellular ROS levels, the lack of enhanced colonization, or migration phenotypes in vitro may indicate that AGC1-knockdown cells have an advantage surviving in the circulation [5]. Although AGC1-KD B16F10 cells do not have reduced baseline ROS levels in vitro, this might be different in vivo. Another indication for increased survival in circulation 


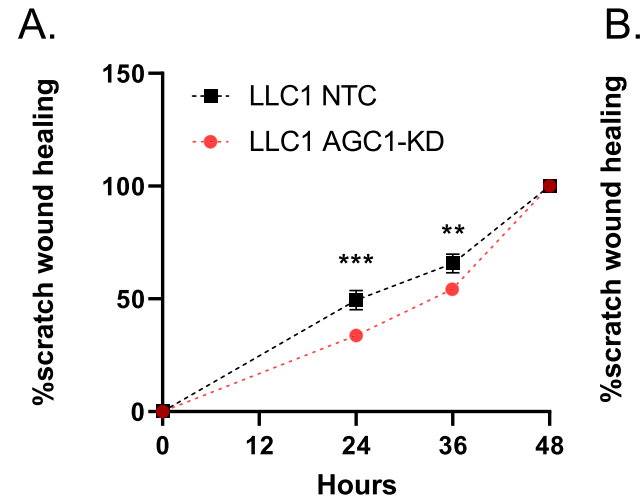

C.

D.
B.

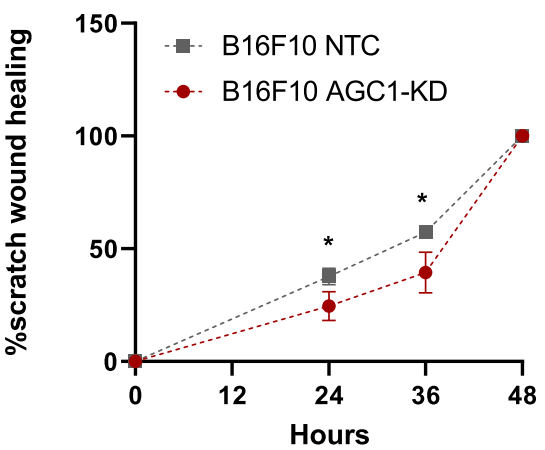

E.
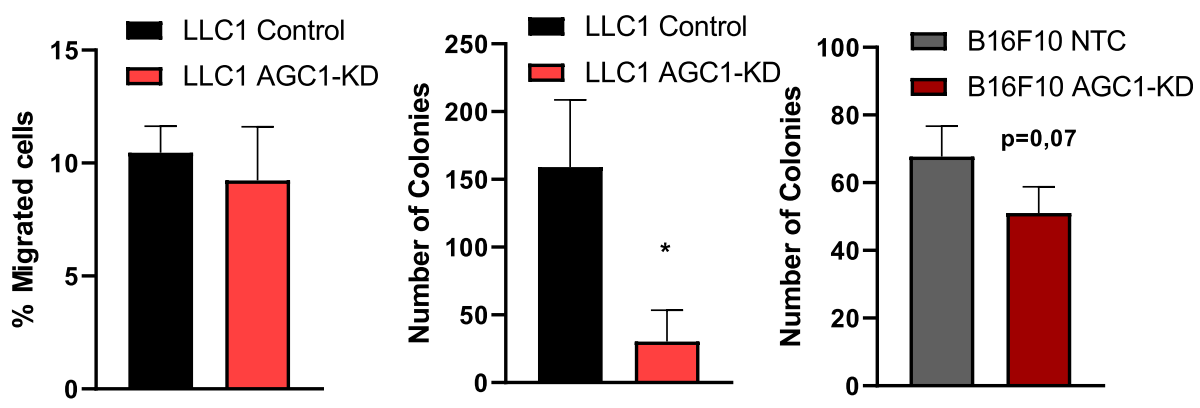

F.

G.
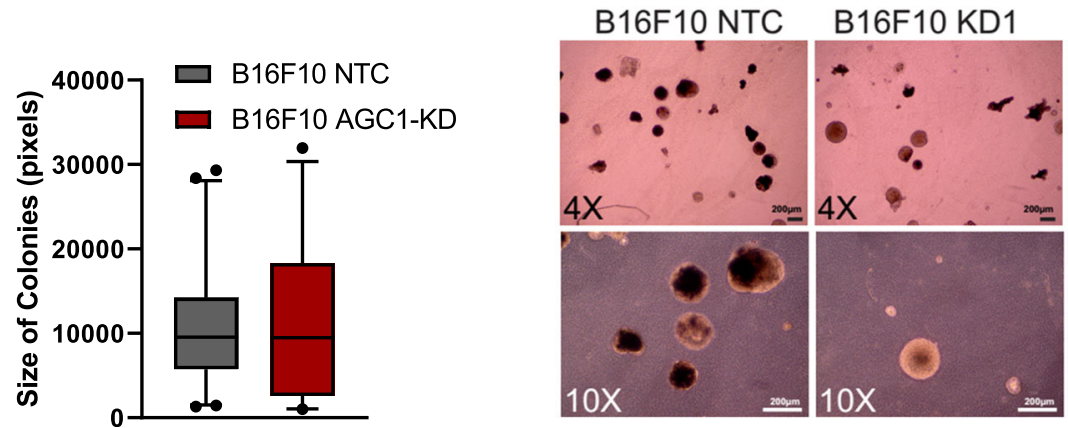

Fig. 4 AGC1-KD cells do not show signs of increased metastatic ability in cell culture. $\mathbf{a}$, b Percent area covered by control (NTC) and AGC1-KD (a) LLC1 or (b) B16F10 cells over the course of 24,36 , and $48 \mathrm{~h}$ following the scratching $(n=5)$. c Percent of control (NTC) and AGC1-KD cells migrated to the bottom wells of the trans-well after $48 \mathrm{~h}(n=3)$. $\mathbf{d}$-e The number of spheres 14 days after seeding of 2.000 control (NTC) and AGC1-KD LLC1 and B16F10 cells, counted by bright field microscopy $(n=3)$. $\mathbf{f}$ Average colony sizes of B16F10 cells from the sphere formation assay in e. $\mathbf{g}$ Representative picture of spheres from B16F10 cells. All experiments denote mean \pm SDs. Significance levels: ${ }^{*} p \leq 0.05$, ** $p \leq 0.01$, *** $p \leq 0.001$

upon AGC1-KD is that AGC1-KD cells yield greater number of individual tumor loci than control cells, while sizes of these tumors were not significantly different. In addition, markers of proliferation and apoptosis stain similarly in the metastatic regions of both AGC1-KD and control tumors. These findings suggest that AGC1KD cells may survive better in the circulation and form greater number of colonies in the lungs compared to control cells. Alternatively, AGC1-KD cells confer an advantage in tumor initiation, and distinguishing these differences will be an interesting topic for future study.

B16F10 and LLC1 cells can metastasize to non-lung tissues including the liver and kidney. Because metastases to these sites are rare, we were unable to determine in a statistically meaningful manner whether AGC1-KD cells metastasize to these tissues more than control cells. Thus, it is possible that the increased metastasis phenotype observed in AGC1-KD tumors is lung-specific. 

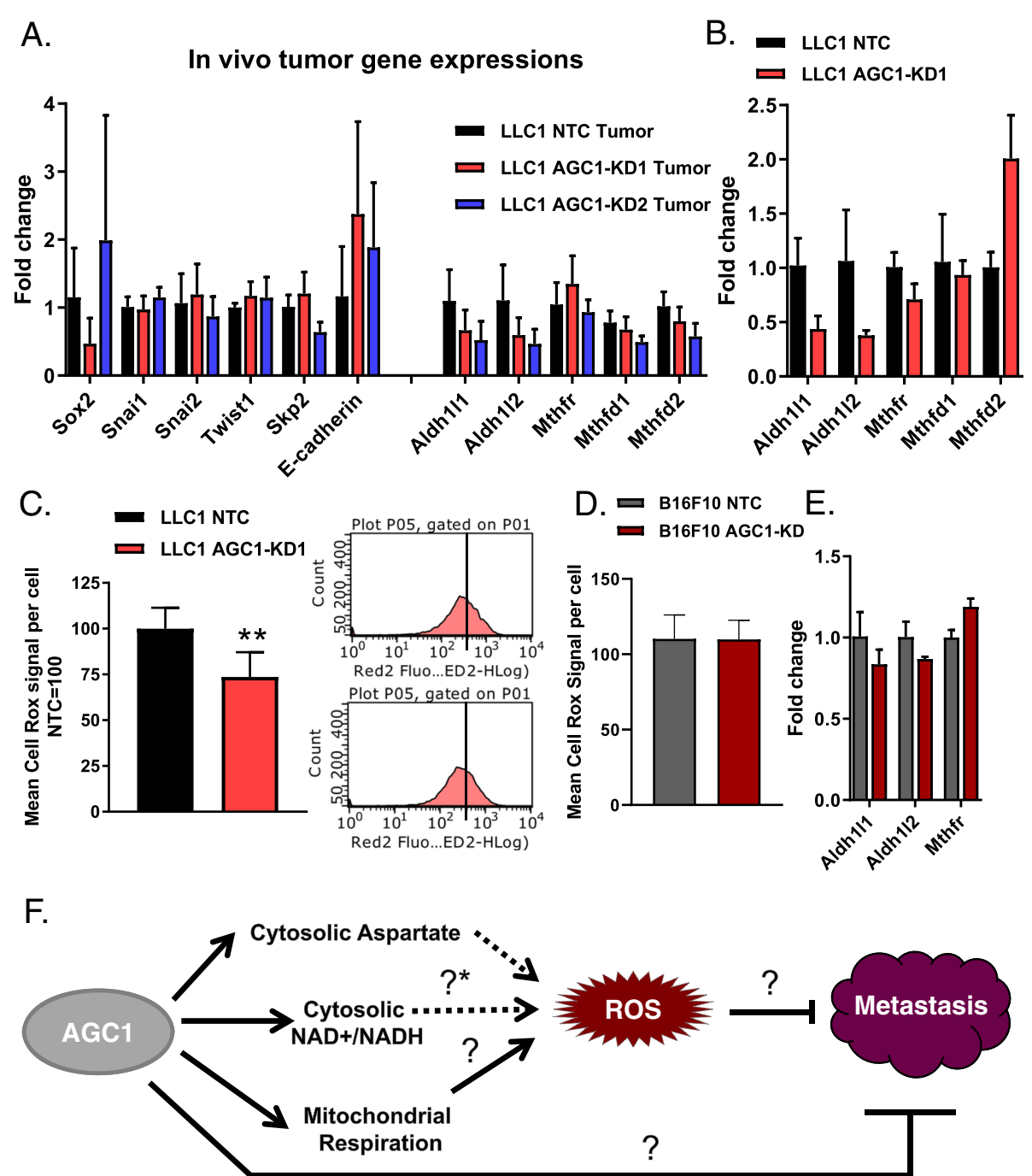

Fig. 5 AGC1-KD LLC1 cells deregulate folate metabolism (genes) and have lower levels of ROS. a mRNA levels of EMT markers and folate pathway genes in control (NTC), AGC1-KD (KD1, sh911 and KD2, sh908) LLC1 tumors in vivo ( $n \geq 6$ ). $\mathbf{b}$ mRNA levels of folate pathway genes in control (NTC), AGC1-KD1 LLC1 cells in vitro $(n=3)$. c (left) Mean CellRox signal per well in AGC1-KD and control (NTC) LLC1 cells; normalized to controls $(n=3)$. (right) Histograms of one representative well from both control (NTC) and AGC1-KD LLC1 cells showing CellRox signal measured via flow cytometry. d Mean CellRox signal per well in AGC1-KD and control (NTC) B16F10 cells; normalized to controls ( $n=3$ ). e mRNA levels of folate pathway genes in control (NTC), AGC1-KD1 B16F10 cells in vitro $(n=3)$. $\mathbf{f}$ Schematic demonstration of potential connections among AGC1 expression, intracellular ROS levels and metastasis. [?*] is referring to the potential changes in folate pathway upon AGC1 expression. All experiments denote mean \pm SDs. Significance levels: ${ }^{*} p \leq 0.05,{ }^{* *} p \leq 0.01,{ }^{* * *} p \leq 0.001$

While the lung environment may somehow be more suitable for AGC1-KD cells to successfully initiate tumors than it is for control cells, if reduced AGC1 promotes survival in circulation it would be expected to also increase metastases in other sites.

Several metabolites including pyruvate, proline, and asparagine were also reported to be involved in the metastasis process [7-9]. However, we failed to detect any increase in these metabolites in vivo in AGC1KD tumors during our previous study that could reflect such metastatic phenotype [15]. Of note, those studies mainly focused on breast cancer metastasis, and lung cancer and melanoma cells may require different metabolic pathways to form metastasis.

Although cells lacking AGC1 did not show signs of apoptosis in standard media conditions, they do proliferate slightly slower than control cells because AGC1 is an important mediator of the malate-aspartate shuttle, cytosolic aspartate levels, nucleotide synthesis, cytosolic $\mathrm{NAD}^{+} / \mathrm{NADH}$ ratio, and mitochondrial $\mathrm{NADH}$ levels [15]. Higher MCT1 expression and increased lactate consumption are correlated with efficient metastasis [6]. We previously observed reduced $\mathrm{NAD}^{+} / \mathrm{NADH}$ and pyruvate/lactate ratios in AGC1-KD cells [15]. These 
may contribute to increased lactate uptake in AGC1-KD cells in vivo, perhaps contributing to a metastatic phenotype in a manner that is similar to that reported in high MCT1-expressing cells. Nevertheless, how AGC1, aspartate transport, and/or the $\mathrm{NAD}^{+} / \mathrm{NADH}$ ratio might be linked to folate metabolism or lactate uptake needs to be studied further. Because the metastatic phenotype is not observed in vitro, we were limited in this study to further examine the mechanistic explanations of why AGC1-KD induces metastasis. However, these findings lay the groundwork for future in vivo studies investigating metabolic requirements of cancer metastasis and how alterations in mitochondrial metabolism may have adverse effects on tumor growth and metastasis.

\section{Supplementary Information}

The online version contains supplementary material available at https://doi. org/10.1186/s40170-020-00232-7.

Additional file 1: Figure S1. AGC1-knockdown in B16F10 impairs cell proliferation and increase lung metastasis (A) (left) Proliferation rate of AGC1-KD and control (NTC) B16F10 cells in doublings/day, cultured in DMEM without pyruvate $(n=3)$ means \pm SDs are shown. (right) Representative pictures of B16F10 cells 64 hours after seeding for the proliferation assay. (B) Pictures of lungs with metastasis harvested from mice 16 days after control and AGC1-KD2 (sh908) B16F10 cells were injected intravenously. (related to Fig. 3)

Additional file 2: Figure S2. Expression of apoptosis and proliferation markers are comparable in metastatic AGC1-KD and metastatic control B16F10 tumors. Representative low-magnification images from the IHC stainings of cleaved caspase 3 (CC3) and PCNA of the metastatic regions from B16F10 tumors. Cleaved Caspase 3 protein (CC3) was used as apoptosis marker. PCNA protein were used as proliferation marker. Images were taken at $4 \mathrm{X}$ magnification. (related to Fig. 3)

Additional file 3: Figure S3. Levels of metabolites and inflammatory cytokines in AGC1-knockdown tumors (A) Relative mRNA expressions of indicated genes in control (NTC) and AGC1-KD1 LLC1 tumors in vivo ( $n=$ 6 ), means \pm SDs are shown. (B-C) Relative mRNA expressions of indicated genes in control (NTC) and AGC1-KD1 (G) LLC1 and (H) B16F10 cells in vitro $(n=3)$, means \pm SDs are shown. Gene names; IL6: Interleukine-6, TNFa:Tumor necrosis factor alpha, IL 1 b:Interleukine1-beta, MCP1:Monocyte chemoattractant protein-1, IL4: Interleukine-4, IL13:Interleukine-13, IL10:Interleukine-10, GM-CSF :Granulocyte-macrophage colony-stimulating factor, and TGFb:Transforming growth factor beta. (related to Fig. 5)

\section{Abbreviations}

AGC1/2: Mitochondrial aspartate-glutamate carriers 1/2; CC3: Cleaved caspase3; EMT: Epithelial-to-mesenchymal transition; GM-CSF: Granulocytemacrophage colony-stimulating factor; KD: Knockdown; NAC: Nacetylcysteine; $\mathrm{NAD}^{+} / \mathrm{NADH}$ : Nicotinamide adenine dinucleotide; ROS: Reactive oxygen species; TCA: Tricarboxylic acid cycle; TGF- $\beta$ : Transforming growth factor beta

\section{Acknowledgements}

We acknowledge the support of DK-MCD, NAWI Graz, constructive discussions with Elke Stadelmeyer, and the technical support of Kathi Walter, Thomas Schreiner, Wolfgang Krispel, and Silvia Schauer. We thank the Histopathology Core at the Koch Institute's Robert A. Swanson Biotechnology Center for technical support.

\section{Authors' contributions}

H.F.A designed and performed the majority of the experiments. P.W.V performed intravenous injections of B16F10 cells and their IHC staining. H.H. conducted bioinformatics analysis. J.F. and M.P. performed cell migration and sphere formation assays. D.R.S conducted IHC imaging of LLC1 tumors. G.H. and M.G.V.H provided substantial guidance and shared lab space and equipment for histology experiments. H.F.A and J.G.B-S constructed the study, and H.F.A wrote the manuscript with significant input from M.G.V.H and P.W.V. The authors read and approved the final manuscript.

\section{Funding}

This work was funded by the Austrian Science Fund FWF SFB LIPTOX F3018, P27108, and W1226 DK "Metabolic and Cardiovascular Disease". M.G.V.H. acknowledges support from the Lustgarten Foundation, SU2C, the Ludwig Center at MIT, the MIT Center for Precision Cancer Medicine, and the NCI (P30 CA1405141, R01 CA168653, R35CA242379). M.G.V.H. is also an HHMI faculty scholar. H.F.A was supported by Austrian Marshall Plan Scholarship. P.W.V. was supported by the ERC LipoCHEX (340896) and the FWF standalone grant LipoLUNG (P 30968). D.R.S. was funded by Harvard Catalyst (National Center for Advancing Translational Sciences, National Institutes of Health Award KL2 TR002542).

\section{Availability of data and materials}

The authors declare that all data supporting the findings of this study are available within the article and its supplementary information files and from the first and corresponding author upon reasonable request. Contact: hfalkan@gmail.com or juliane.bogner-strauss@stmk.gv.at

\section{Ethics approval and consent to participate}

The animal study was approved by the institutional ethics committee, and experiments were performed according to the guidelines of the Austrian Federal Ministry of Science and Research. Experiment licenses were granted under BMWF-66.007/0026-WFN/3b/2015 and BMWF-66.007/0008-WFN/3b/ 2016. In vivo, CB-839 experiments were approved by the MIT Committee on Animal Care (IACUC).

\section{Consent for publication}

Not applicable.

\section{Competing interests}

The authors declare no competing interests.

\section{Author details}

'Institute of Biochemistry, Graz University of Technology, Humboldtstrasse 46/III, 8010 Graz, Austria. ${ }^{2}$ The Koch Institute for Integrative Cancer Research and Department of Biology, Massachusetts Institute of Technology,

Cambridge, MA 02139, USA. 'Diagnostic and Research Institute of Pathology, Medical University of Graz, Neue Stiftingtalstrasse 6, 8010 Graz, Austria.

${ }^{4}$ Dana-Farber Cancer Institute, Boston, MA 02115, USA. Institute of Bioinformatics, Biocenter, Medical University of Innsbruck, Innrain 80, 6020 Innsbruck, Austria. ${ }^{6}$ Division of Oncology, Department of Internal Medicine, Medical University of Graz, Auenbruggerplatz 15, 8036 Graz, Austria. ${ }^{7}$ Department of Radiation Oncology, Beth Israel Deaconess Medical Center, Boston, MA, USA. ${ }^{8}$ Harvard Medical School, Boston, MA, USA. ${ }^{9}$ Department of Experimental Therapeutics, UT MD Anderson Cancer Center, Houston, USA.

${ }^{10}$ BioTechMed-Graz, Graz, Austria.

Received: 11 May 2020 Accepted: 1 November 2020

Published online: 26 November 2020

References

1. Fendt SM. Metabolic vulnerabilities of metastasizing cancer cells. BMC Biol. 2019:17:54.

2. Elia I, Doglioni G, Fendt SM. Metabolic hallmarks of metastasis formation. Trends Cell Biol. 2018;28:673-84.

3. Peiris-Pages M, Martinez-Outschoorn UE, Sotgia F, Lisanti MP. Metastasis and oxidative stress: are antioxidants a metabolic driver of progression? Cell Metab. 2015;22:956-8.

4. Le Gal K, Ibrahim MX, Wiel C, Sayin VI, Akula MK, Karlsson C, Dalin MG, Akyurek LM, Lindahl P, Nilsson J, Bergo MO. Antioxidants can increase melanoma metastasis in mice. Sci Transl Med. 2015;7:308re8.

5. Piskounova E, Agathocleous M, Murphy MM, Hu Z, Huddlestun SE, Zhao Z, Leitch AM, Johnson TM, Deberardinis RJ, Morrison SJ. Oxidative stress inhibits distant metastasis by human melanoma cells. Nature. 2015;527: 186-91. 
6. Tasdogan A, Faubert B, Ramesh V, Ubellacker JM, Shen B, Solmonson A, Murphy MM, Gu Z, Gu W, Martin M, Kasitinon SY, Vandergriff T, Mathews TP, Zhao Z, Schadendorf D, Deberardinis RJ, Morrison SJ. Metabolic heterogeneity confers differences in melanoma metastatic potential. Nature. 2020;577:115-20.

7. Elia I, Broekaert D, Christen S, Boon R, Radaelli E, Orth MF, Verfaillie C, Grunewald TGP, Fendt SM. Proline metabolism supports metastasis formation and could be inhibited to selectively target metastasizing cancer cells. Nat Commun. 2017:8:15267.

8. Elia I, Rossi M, Stegen S, Broekaert D, Doglioni G, Van Gorsel M, Boon R, Escalona-Noguero C, Torrekens S, Verfaillie C, Verbeken E, Carmeliet G, Fendt SM. Breast cancer cells rely on environmental pyruvate to shape the metastatic niche. Nature. 2019:568:117-21.

9. Knott SRV, Wagenblast E, Khan S, Kim SY, Soto M, Wagner M, Turgeon MO, Fish L, Erard N, Gable AL, Maceli AR, Dickopf S, Papachristou EK, D'santos CS, Carey LA, Wilkinson JE, Harrell JC, Perou CM, Goodarzi H, Poulogiannis G, Hannon GJ. Asparagine bioavailability governs metastasis in a model of breast cancer. Nature. 2018;554:378-81.

10. Gui DY, Sullivan LB, Luengo A, Hosios AM, Bush LN, Gitego N, Davidson SM, Freinkman E, Thomas CJ, Vander Heiden MG. Environment dictates dependence on mitochondrial complex I for NAD+ and aspartate production and determines cancer cell sensitivity to metformin. Cell Metab. 2016:24:716-27.

11. Garcia-Bermudez J, Baudrier L, La K, Zhu XG, Fidelin J, Sviderskiy VO, Papagiannakopoulos T, Molina H, Snuderl M, Lewis CA, Possemato RL, Birsoy K. Aspartate is a limiting metabolite for cancer cell proliferation under hypoxia and in tumours. Nat Cell Biol. 2018;20:775-81.

12. Sullivan LB, Luengo A, Danai LV, Bush LN, Diehl FF, Hosios AM, Lau AN, Elmiligy S, Malstrom S, Lewis CA, Vander Heiden MG. Aspartate is an endogenous metabolic limitation for tumour growth. Nat Cell Biol. 2018;20:782-8.

13. Birsoy K, Wang T, Chen WW, Freinkman E, Abu-Remaileh M, Sabatini DM. An essential role of the mitochondrial electron transport chain in cell proliferation is to enable aspartate synthesis. Cell. 2015;162:540-51.

14. Sullivan LB, Gui DY, Hosios AM, Bush LN, Freinkman E, Vander Heiden MG. Supporting aspartate biosynthesis is an essential function of respiration in proliferating cells. Cell. 2015;162:552-63.

15. Alkan HF, Walter KE, Luengo A, Madreiter-Sokolowski CT, Stryeck S, Lau AN, Al-Zoughbi W, Lewis CA, Thomas CJ, Hoefler G, Graier WF, Madl T, Vander Heiden MG, Bogner-Strauss JG. Cytosolic aspartate availability determines cell survival when glutamine is limiting. Cell Metab. 2018;28(706-720):e6.

16. Alkan HF, Bogner-Strauss JG. Maintaining cytosolic aspartate levels is a major function of the TCA cycle in proliferating cells. Mol Cell Oncol. 2019;6: e1536843.

17. Altman DG, Lausen B, Sauerbrei W, Schumacher M. Dangers of using "optimal" cutpoints in the evaluation of prognostic factors. J Natl Cancer Inst. 1994;86:829-35.

18. Schwarzenbacher D, Klec C, Pasculli B, Cerk S, Rinner B, Karbiener M, Ivan C, Barbano R, Ling H, Wulf-Goldenberg A, Stanzer S, Rinnerthaler G, Stoeger H, Bauernhofer T, Haybaeck J, Hoefler G, Jahn SW, Parrella P, Calin GA, Pichler M. MiR-1287-5p inhibits triple negative breast cancer growth by interaction with phosphoinositide 3-kinase CB, thereby sensitizing cells for PI3Kinase inhibitors. Breast Cancer Res. 2019;21:20.

19. Al-Zoughbi W, Pichler M, Gorkiewicz G, Guertl-Lackner B, Haybaeck J, Jahn SW, Lackner C, Liegl-Atzwanger B, Popper H, Schauer S, Nusshold E, Kindt ASD, Trajanoski Z, Speicher MR, Haemmerle G, Zimmermann R, Zechner R, Vesely PW, Hoefler G. Loss of adipose triglyceride lipase is associated with human cancer and induces mouse pulmonary neoplasia. Oncotarget. 2016; 7:33832-40.

20. Thangaratnarajah C, Ruprecht JJ, Kunji ER. Calcium-induced conformational changes of the regulatory domain of human mitochondrial aspartate/ glutamate carriers. Nat Commun. 2014;5:5491.

21. Hart IR. The selection and characterization of an invasive variant of the B16 melanoma. Am J Pathol. 1979;97:587-600.

22. Gao H, Teng C, Huang W, Peng J, Wang C. SOX2 promotes the epithelial to mesenchymal transition of esophageal squamous cells by modulating slug expression through the activation of STAT3/HIF-alpha signaling. Int J Mol Sci. 2015;16:21643-57.

23. Yang Q, Huang J, Wu Q, Cai Y, Zhu L, Lu X, Chen S, Chen C, Wang Z. Acquisition of epithelial-mesenchymal transition is associated with Skp2 expression in paclitaxel-resistant breast cancer cells. Br J Cancer. 2014;110: 1958-67.

24. Singh S, Brocker C, Koppaka V, Chen Y, Jackson BC, Matsumoto A, Thompson DC, Vasiliou V. Aldehyde dehydrogenases in cellular responses to oxidative/electrophilic stress. Free Radic Biol Med. 2013;56:89-101.

25. Blomberg OS, Spagnuolo L, De Visser KE. Immune regulation of metastasis: mechanistic insights and therapeutic opportunities. Dis Model Mech. 2018; 11:dmm036236.

26. Janssen LME, Ramsay EE, Logsdon CD, Overwijk WW. The immune system in cancer metastasis: friend or foe? J Immunother Cancer. 2017;5:79.

27. Neagu M, Constantin C, Popescu ID, Zipeto D, Tzanakakis G, Nikitovic D, Fenga C, Stratakis CA, Spandidos DA, Tsatsakis AM. Inflammation and metabolism in cancer cell-mitochondria key player. Front Oncol. 2019;9:348.

\section{Publisher's Note}

Springer Nature remains neutral with regard to jurisdictional claims in published maps and institutional affiliations.

Ready to submit your research? Choose BMC and benefit from:

- fast, convenient online submission

- thorough peer review by experienced researchers in your field

- rapid publication on acceptance

- support for research data, including large and complex data types

- gold Open Access which fosters wider collaboration and increased citations

- maximum visibility for your research: over $100 \mathrm{M}$ website views per year

At BMC, research is always in progress.

Learn more biomedcentral.com/submissions 